\title{
Seasonal variations in biomass and nutrients in Eichhornia crassipes (Martius) Solms-Laubach at Biratnagar, Nepal
}

\author{
Umesh Koirala*, Sasinath Jha, Bhabindra Niroula and Bindu Pokharel (Bhattarai) \\ Department of Botany \\ Post Graduate Campus, Tribhuvan University, Biratnagar, Nepal \\ *E-mail: umesh6977@hotmail.com
}

\begin{abstract}
In a derelict depression retaining water throughout the year, the importance value index of E. crassipes ranged between 23.06 (March) and 146.4 (January), whereas biomass was between 4.16 (March) and $1280.8 \mathrm{~g} / \mathrm{m}^{2}$ (November). The net primary productivity of the plant along with associated species was $2058.79 \mathrm{~g} / \mathrm{m}^{2} / \mathrm{yr}$. On the annual basis, percentage concentration of different nutrients in different plant parts occurred in the order: (i) $\mathrm{N}$ : stem (1.69) > whole leaf (1.65) > root (1.56) > pseudolamina (1.08); (ii) P: root (1.33) > whole leaf and pseudolamina (0.5) > stem (0.49); and (iii) K: pseudolamina $(2.42)>$ stem and whole leaf $(2.03)>\operatorname{root}(1.41)$. Habitat water had maximum values of total nitrogen $(8.25 \mathrm{mg} / \mathrm{l})$, nitrite $(0.05 \mathrm{mg} / \mathrm{l})$ and potassium $(19.7 \mathrm{mg} / \mathrm{l})$ in winter; $\mathrm{pH}(6.4)$, conductivity $(586 \mathrm{ds} / \mathrm{m})$, ammonia $(1.16 \mathrm{mg} / \mathrm{l})$ and nitrate $(0.15 \mathrm{mg} / \mathrm{l})$ in summer; and temperature $\left(31.2^{0} \mathrm{C}\right)$, turbidity $(95 \mathrm{NTU})$ and total dissolved solids $(650 \mathrm{mg} / \mathrm{l})$ in rainy season. The sediment (mud) of the habitat was silty-clay loam with annual averages of $\mathrm{pH} 5$, nitrogen $0.25 \%$, phosphorus $0.0014 \%$, potassium $0.076 \%$, organic carbon $3.95 \%$ and $\mathrm{C} / \mathrm{N}$ ratio $16: 1$.
\end{abstract}

Key words: Water-hyacinth, IVI, biomass, diversity indices, productivity, nutrients

\section{Introduction}

Known as water-hyacinth and a native of neotropics, E. crassipes (family Pontederiaceae) is a free-floating fresh water perennial herb found in a large variety of aquatic habitats and is often dominant over large areas. The plant usually propagates and multiplies vegetatively. Parija (1934) observed seven-fold increase in water-hyacinth spread in 50 days and Penfound and Earle (1948) reported that the edge of water-hyacinth mat extended by $60 \mathrm{~cm}$ every month. The growth of water-hyacinth does not appear to be density-dependent since the mat could rapidly grow so dense and thick and often so strong as to allow man to walk over it. The plants in dense growth obstruct water flow in irrigation channels; interfere with navigation and hydroelectric power generation. Water-hyacinth suppresses the growth of phytoplanktons and submerged plants. Its cover provides obnoxious smell, coloring matter and suspended particulate matter in water. It increases carbon dioxide concentration, and decreases clarity and dissolved oxygen content in water. It is rated as the world's most troublesome weed that has defied all efforts (mechanical, biological and chemical) of man to control it. Nevertheless, the plant has potentials to be used as fodder, manure, as a source of methane and alcohol, for purifying water and also for its beautiful lilac violet flowers for decoration (Cook, 1996), so that the plant should be groomed as a useful resource rather treated as a nuisance. In this context, the present study deals with monthly (January-December, 2011) variations in IVI, biomass, and species diversity indices in E. crassipes association along with variations in concentration of $\mathrm{N}, \mathrm{P}$ and $\mathrm{K}$ in different parts of the plant as well as some physico-chemical properties of water and sediment (mud) of its habitat. 
The study site is an elongated depression (area $200 \times 20 \mathrm{~m}$ ) surrounded by agricultural land and situated south to Post Graduate Campus at $1 \mathrm{~km}$ distance. It is fed by rain water as well as runoff water from the surrounding agricultural land, and depth of water in the centre of the depression was recorded 150 and $45 \mathrm{~cm}$ in rainy and summer season, respectively during the present study. In general, Biratnagar (lat. N 26 20'; long. E $87^{0} 16^{\prime}$; altitude $72 \mathrm{~m}$, msl) has tropical and monsoonic climate with three distinct seasons viz, rainy (mid-June to October), winter (November to February), and summer (March to mid-June). Average meteorological data indicate 1225,5 , and $188 \mathrm{~mm}$ rainfall; $25,10.4$ and $19.6^{\circ} \mathrm{C}$ minimum air temperature; 32.2, 25 and $33.6^{\circ} \mathrm{C}$ maximum air temperature; and $6,3.8$ and $7.3 \mathrm{~km} / \mathrm{h}$ wind speed during rainy, winter and summer season, respectively for the last five years.

\section{Materials and Methods}

Individuals of E. crassipes and the associated species of the shore area $(15 \mathrm{~cm}$ to $1 \mathrm{~m}$ water depth) were sampled by laying ten quadrats of $50 \mathrm{~cm} \times 50 \mathrm{~cm}$ size randomly for determination of IVI, diversity indices, and biomass at monthly intervals (first week of each month). The IVI was calculated as the sum of relative frequency, relative density and relative cover (Curtis \& McIntosh, 1951), and diversity indices were determined as per the method of Simpson (1949), and Shannon and Weaver (1949). The net primary productivity (NPP) was calculated by summing the positive increments in dry biomass on successive sampling dates (Singh \& Yadava, 1974).

Dried plant and mud samples were analysed for nitrogen by Nessler's reagent method (Willard et al., 1974), phosphorus by chlorostannous-reduced molybdophosphoric blue color method (Furman, 1962), and potassium by flame photometer method (Piper, 1944). The texture of the mud was determined by sieving and weighing method (Piper, 1944), and organic carbon by Walkley and Black's (1934) method. Among the physico-chemical parameters of water samples, $\mathrm{pH}$ was determined by digital $\mathrm{pH}$ meter; temperature by thermometer; conductivity by electrical conductivity meter (Willard et al., 1974); turbidity (nephelometric method), phosphorus (vandomolybdophophoric acid colorimetric method), total nitrogen (macroKjeldahl method), nitrate and nitrite (UV spectrophotometric method), and potassium (atomic absorption spectrophotometric method) by APHA's (1990) methods; and total dissolved solids by the method of U.S. Geological Survey.

\section{Results and Discussion}

\section{IVI, diversity indices, biomass and productivity}

The IVI of E. crassipes decreased from February to March, increased remarkably between April and May, decreased in June and July, and thereafter increased gradually from August reaching the highest value (146.4) in January (Table 1). Species next to E. crassipes in IVI were Alternanthera philoxeroides and Spirodela polyrhiza in January, and S. polyrhiza and Potamogeton crispus in February. Nitella mucronatus and A. philoxeroides had greater IVI than E. crassipes in March. Species next to E. crassipes in IVI were A. philoxeroides and $S$. polyrhiza in April and May, A. philoxeroides and Lemna aequinoctialis in June, A. philoxeroides and S. polyrhiza from July to October, and S. polyrhiza and L. aequinoctialis in November and December. McCann (2000) has opined that as real populations are variable, it is possible that the persistence of complex communities depends to some degree on population fluxes (that is, the fairly regular waxing and waning of a population's density). Such background population variability, whether driven by biotic or abiotic processes, can provide species with opportunity to respond differentially to their environment. In turn, these differential responses weaken the destructive potential of competitive exclusion. 
The range of Simpson's diversity index was between 0.65 and 0.81 , whereas Shannon-Weiner's diversity index ranged between 1.35 and 1.85 in December and March, respectfully (Table 1). Both the indices displayed decreasing trend from August to December, increasing trend from January to March, and decreasing-increasing trend between April to July. The increase in the values of diversity indices may be attributed to decline in IVI and biomass of E. crassipes, whereas decreasing-increasing trend may be the result of fluctuation in water level. Decrease in the values of diversity indices between August to December was due to dominance of $E$. crassipes. These findings were in agreement with Krebs (1972) that diversity increases as any particular stress lessens.

Table 1 . Monthly variations in IVI (mean \pm SE; $n=10$ ) and diversity indices in Eichhornia crassipes association.

\begin{tabular}{|c|c|c|c|c|c|c|c|c|c|c|c|c|}
\hline & Jan & Feb & Mar & Apr & May & Jun & Jul & Aug & Sep & Oct & Nov & Dec \\
\hline \multicolumn{13}{|l|}{ IVI } \\
\hline $\begin{array}{l}\text { Alternanthera } \\
\text { philoxeroides }\end{array}$ & $\begin{array}{l}47.44 \\
\pm 2.18\end{array}$ & - & $\begin{array}{l}67.54 \\
\pm 2.60\end{array}$ & $\begin{array}{l}95.35 \\
\pm 3.09\end{array}$ & $\begin{array}{l}41.90 \\
\pm 2.05\end{array}$ & $\begin{array}{l}56.87 \\
\pm 2.38\end{array}$ & $\begin{array}{l}77.41 \\
\pm 2.78\end{array}$ & $\begin{array}{r}79.39 \\
\pm 2.82\end{array}$ & $\begin{array}{l}87.34 \\
\pm 2.95\end{array}$ & $\begin{array}{l}48.13 \\
\pm 2.19\end{array}$ & $\begin{array}{l}27.26 \\
\pm 1.65\end{array}$ & $\begin{array}{l}31.28 \\
\pm 1.77\end{array}$ \\
\hline $\begin{array}{l}\text { Azolla } \\
\text { imbricata }\end{array}$ & $\begin{array}{l}20.57 \\
\pm 1.43 \\
\end{array}$ & $\begin{array}{l}18.88 \\
\pm 1.37 \\
\end{array}$ & $\begin{array}{l}31.02 \\
\pm 1.76 \\
\end{array}$ & $\begin{array}{l}23.96 \\
\pm 1.55 \\
\end{array}$ & $\begin{array}{l}40.83 \\
\pm 2.02 \\
\end{array}$ & $\begin{array}{l}32.29 \\
\pm 1.80 \\
\end{array}$ & $\begin{array}{l}35.54 \\
\pm 1.88 \\
\end{array}$ & $\begin{array}{l}14.26 \\
\pm 1.19 \\
\end{array}$ & $\begin{array}{c}4.65 \\
\pm 0.68 \\
\end{array}$ & - & - & $\begin{array}{c}8.65 \\
\pm .0 .93 \\
\end{array}$ \\
\hline $\begin{array}{l}\text { Colocasia } \\
\text { esculenta }\end{array}$ & - & - & - & - & - & - & $\begin{array}{c}4.98 \\
\pm 0.70\end{array}$ & $\begin{array}{c}9.10 \\
\pm 0.95\end{array}$ & $\begin{array}{c}4.69 \\
\pm 0.68\end{array}$ & - & - & - \\
\hline $\begin{array}{l}\text { Eichhornia } \\
\text { crassipes }\end{array}$ & $\begin{array}{l}146.4 \\
\pm 3.83 \\
\end{array}$ & $\begin{array}{c}109.57 \\
\pm 3.1 \\
\end{array}$ & $\begin{array}{l}23.06 \\
\pm 1.52 \\
\end{array}$ & $\begin{array}{l}101.69 \\
\pm 3.19 \\
\end{array}$ & $\begin{array}{c}137.29 \\
\pm 3.7 \\
\end{array}$ & $\begin{array}{c}107.85 \\
\pm 3.28 \\
\end{array}$ & $\begin{array}{r}93.97 \\
\pm 3.06 \\
\end{array}$ & $\begin{array}{l}98.08 \\
\pm 3.13 \\
\end{array}$ & $\begin{array}{l}106.39 \\
\pm 3.26 \\
\end{array}$ & $\begin{array}{c}135.80 \\
\pm 3.68 \\
\end{array}$ & $\begin{array}{l}135.88 \\
\pm 3.69 \\
\end{array}$ & $\begin{array}{c}140.82 \\
\pm 3.75 \\
\end{array}$ \\
\hline $\begin{array}{l}\text { Euryale } \\
\text { ferox }\end{array}$ & - & $\begin{array}{l}14.18 \\
\pm 1.19 \\
\end{array}$ & $\begin{array}{c}3.53 \\
\pm .0 .59 \\
\end{array}$ & - & - & - & - & - & - & -- & - & - \\
\hline $\begin{array}{l}\text { Lemna } \\
\text { aequinoctialis }\end{array}$ & $\begin{array}{l}42.77 \\
\pm 2.07 \\
\end{array}$ & $\begin{array}{l}23.69 \\
\pm 1.54 \\
\end{array}$ & $\begin{array}{l}32.50 \\
\pm 1.80 \\
\end{array}$ & $\begin{array}{l}31.61 \\
\pm 1.78 \\
\end{array}$ & $\begin{array}{l}38.75 \\
\pm 1.97 \\
\end{array}$ & $\begin{array}{l}54.74 \\
\pm 2.34 \\
\end{array}$ & $\begin{array}{l}36.29 \\
\pm 1.90 \\
\end{array}$ & $\begin{array}{r}33.04 \\
\pm 1.82 \\
\end{array}$ & $\begin{array}{l}30.13 \\
\pm 1.73 \\
\end{array}$ & $\begin{array}{r}10.74 \\
\pm .1 .04 \\
\end{array}$ & $\begin{array}{l}62.89 \\
\pm 2.51 \\
\end{array}$ & $\begin{array}{l}57.39 \\
\pm 2.39 \\
\end{array}$ \\
\hline Lemna sp. & - & - & - & - & - & $\begin{array}{l}15.74 \\
\pm 1.25\end{array}$ & $\begin{array}{l}15.40 \\
\pm 1.24\end{array}$ & - & $\begin{array}{c}4.67 \\
\pm 0.68\end{array}$ & $\begin{array}{l}10.76 \\
\pm 1.04\end{array}$ & $\begin{array}{c}5.01 \\
\pm 0.71\end{array}$ & - \\
\hline $\begin{array}{l}\text { Mikania } \\
\text { micrantha }\end{array}$ & - & - & - & - & - & - & - & - & $\begin{array}{c}4.62 \\
\pm 0.68 \\
\end{array}$ & $\begin{array}{l}25.06 \\
\pm 1.58\end{array}$ & $\begin{array}{c}5.14 \\
\pm 0.72\end{array}$ & - \\
\hline $\begin{array}{l}\text { Nitella } \\
\text { mucronatus }\end{array}$ & - & $\begin{array}{l}36.86 \\
\pm 1.92 \\
\end{array}$ & $\begin{array}{l}92.34 \\
\pm 3.04 \\
\end{array}$ & - & - & - & - & - & - & - & - & $\begin{array}{r}3.80 \\
\pm 0.62 \\
\end{array}$ \\
\hline $\begin{array}{l}\text { Potamogeton } \\
\text { crispus }\end{array}$ & - & $\begin{array}{l}43.95 \\
\pm 2.10 \\
\end{array}$ & $\begin{array}{l}16.71 \\
\pm 1.29 \\
\end{array}$ & - & - & - & - & - & - & - & - & - \\
\hline $\begin{array}{l}\text { Spirodela } \\
\text { polyrhiza }\end{array}$ & $\begin{array}{l}42.82 \\
\pm 2.07 \\
\end{array}$ & $\begin{array}{l}52.87 \\
\pm 2.30 \\
\end{array}$ & $\begin{array}{l}33.30 \\
\pm 1.82 \\
\end{array}$ & $\begin{array}{l}47.39 \\
\pm 2.18 \\
\end{array}$ & $\begin{array}{l}41.23 \\
\pm 2.03 \\
\end{array}$ & $\begin{array}{l}32.51 \\
\pm 1.80 \\
\end{array}$ & $\begin{array}{l}36.41 \\
\pm 1.91 \\
\end{array}$ & $\begin{array}{l}66.13 \\
\pm 2.57 \\
\end{array}$ & $\begin{array}{l}57.51 \\
\pm 2.40 \\
\end{array}$ & $\begin{array}{l}69.51 \\
\pm 2.64 \\
\end{array}$ & $\begin{array}{l}63.82 \\
\pm 2.53 \\
\end{array}$ & $\begin{array}{l}58.06 \\
\pm 2.41 \\
\end{array}$ \\
\hline \multicolumn{13}{|c|}{ Diversity indices } \\
\hline $\begin{array}{l}\text { Simpson's } \\
\text { index }\end{array}$ & 0.69 & 0.79 & 0.81 & 0.74 & 0.71 & 0.77 & 0.79 & 0.75 & 0.75 & 0.71 & 0.70 & 0.65 \\
\hline $\begin{array}{l}\text { Shannon- } \\
\text { Weiner's index }\end{array}$ & 1.37 & 1.73 & 1.85 & 1.45 & 1.43 & 1.62 & 1.69 & 1.53 & 1.47 & 1.42 & 1.40 & 1.35 \\
\hline
\end{tabular}

The biomass of E. crassipes decreased from December to March, increased drastically in April, and thereafter exhibited decreasing-increasing trend until November (Table 2). Species next to E. crassipes in biomass value was A. philoxeroides from April to January, whereas $N$. mucronatus dominated the biomass value in March. The productivity of E. crassipes association was $2058.79 \mathrm{~g} / \mathrm{m}^{2} / \mathrm{yr}$ in which percentage contributions of the major species were in the order: E. crassipes $(62.54)>A$. philoxeroides $(30.42)>S$. polyrhiza $(0.8)>$ L. aequinoctialis $(0.05)$. Other species present in the association had $6.19 \%$ contributions to the net productivity. Earlier, Das (1968) observed the rate of production of E. crassipes to vary from 3.48 to $9.98 \mathrm{~g} / \mathrm{m}^{2} /$ day in different months, and Bellamy (1967) reported a similar value $\left(9.7 \mathrm{~g} / \mathrm{m}^{2} /\right.$ day $)$ from Assam, 
India. Mitsch and Gosselink (2000) have stated that NPP is between $100-500 \mathrm{~g} / \mathrm{m}^{2} / \mathrm{yr}$ in lownutrient wetlands, whereas in high-nutrient wetlands NPP is between $1000-4000 \mathrm{~g} / \mathrm{m}^{2} / \mathrm{yr}$. According to Kroll et al. (1997), shallow water conditions usually result in the highest productivity whereas deep-water conditions offer the least potential for both emergent plants and invading, undesirable free-floating plants.

Table 2. Monthly variations in biomass $\left(\mathrm{g} / \mathrm{m}^{2}\right)$ (mean $\left.\pm \mathrm{SE} ; \mathrm{n}=10\right)$ in Eichhornia crassipes association.

\begin{tabular}{|c|c|c|c|c|c|c|c|c|c|c|c|c|}
\hline Species & Jan & Feb & Mar & Apr & May & Jun & Jul & Aug & Sep & Oct & Nov & Dec \\
\hline $\begin{array}{l}\text { Alternanthera } \\
\text { philoxeroides }\end{array}$ & $\begin{array}{l}36.3 \\
\pm 1.9 \\
\end{array}$ & - & $\begin{array}{l}94.0 \\
\pm 3.06\end{array}$ & $\begin{array}{l}264 \\
\pm 5.14\end{array}$ & $\begin{array}{l}19.0 \\
\pm 1.38 \\
\end{array}$ & $\begin{array}{l}264.0 \\
\pm 5.14 \\
\end{array}$ & $\begin{array}{l}575.2 \\
\pm 7.58\end{array}$ & $\begin{array}{l}575 \\
\pm 7.58 \\
\end{array}$ & $\begin{array}{l}268 \\
\pm 5.18\end{array}$ & $\begin{array}{l}84.00 \\
\pm 2.90 \\
\end{array}$ & $\begin{array}{l}36.00 \\
\pm 1.90\end{array}$ & $\begin{array}{l}22.0 \\
\pm 1.48\end{array}$ \\
\hline $\begin{array}{l}\text { Azolla } \\
\text { imbricata }\end{array}$ & $\begin{array}{l}0.26 \\
\pm 0.51\end{array}$ & $\begin{array}{l}0.02 \\
\pm 0.04\end{array}$ & $\begin{array}{l}0.27 \\
\pm 0.52\end{array}$ & $\begin{array}{l}0.02 \\
\pm 0.14\end{array}$ & $\begin{array}{l}0.02 \\
\pm 0.14\end{array}$ & $\begin{array}{l}0.56 \\
\pm 0.24\end{array}$ & $\begin{array}{l}0.05 \\
\pm 0.07\end{array}$ & $\begin{array}{l}0.7 \\
\pm 0.26\end{array}$ & $\begin{array}{l}0.48 \\
\pm 0.22\end{array}$ & - & - & $\begin{array}{l}0.26 \\
\pm 0.16\end{array}$ \\
\hline $\begin{array}{l}\text { Colocasia } \\
\text { esculenta }\end{array}$ & - & - & - & - & - & - & $\begin{array}{l}0.60 \\
\pm 0.24\end{array}$ & $\begin{array}{l}1.0 \\
\pm 0.32 \\
\end{array}$ & $\begin{array}{l}0.80 \\
\pm 0.28\end{array}$ & - & - & - \\
\hline $\begin{array}{l}\text { Eichhornia } \\
\text { crassipes }\end{array}$ & $\begin{array}{l}351.2 \\
\pm 5.93\end{array}$ & $\begin{array}{l}130 \\
\pm 3.60\end{array}$ & $\begin{array}{l}4.16 \\
\pm 0.64\end{array}$ & $\begin{array}{l}642 \\
\pm 8.01\end{array}$ & $\begin{array}{l}586 \\
\pm 7.65\end{array}$ & $\begin{array}{l}830.0 \\
\pm 9.11\end{array}$ & $\begin{array}{l}890 \\
\pm 9.43\end{array}$ & $\begin{array}{l}814 \\
\pm 9.02\end{array}$ & $\begin{array}{l}610 \\
\pm 7.81\end{array}$ & $\begin{array}{l}1099 \\
\pm 10.49\end{array}$ & $\begin{array}{l}1280 \\
\pm 11.32\end{array}$ & $\begin{array}{l}935.5 \\
\pm 9.67\end{array}$ \\
\hline $\begin{array}{l}\text { Euryale } \\
\text { ferox }\end{array}$ & - & $\begin{array}{l}0.93 \\
\pm 0.30 \\
\end{array}$ & $\begin{array}{l}2.43 \\
\pm 0.48 \\
\end{array}$ & $\begin{array}{l}0.02 \\
\pm 0.14 \\
\end{array}$ & - & - & - & - & - & - & - & - \\
\hline $\begin{array}{l}\text { Lemna } \\
\text { aequinoctialis }\end{array}$ & $\begin{array}{l}1.44 \\
\pm 0.38\end{array}$ & $\begin{array}{l}0.37 \\
\pm 0.19\end{array}$ & $\begin{array}{l}0.40 \\
\pm 0.20\end{array}$ & - & - & $\begin{array}{l}0.02 \\
\pm 0.14\end{array}$ & $\begin{array}{l}0.02 \\
\pm 0.14\end{array}$ & $\begin{array}{l}0.02 \\
\pm 0.14\end{array}$ & $\begin{array}{l}0.24 \\
\pm 0.15\end{array}$ & $\begin{array}{l}0.02 \\
\pm 0.14\end{array}$ & $\begin{array}{l}0.61 \\
\pm 0.25\end{array}$ & $\begin{array}{l}1.04 \\
\pm 0.32 \\
\end{array}$ \\
\hline Lemna sp. & - & - & - & - & - & $\begin{array}{l}0.02 \\
\pm 0.14\end{array}$ & $\begin{array}{l}0.02 \\
\pm 0.14\end{array}$ & - & $\begin{array}{l}0.21 \\
\pm 0.14\end{array}$ & $\begin{array}{l}22 \\
\pm 0.15\end{array}$ & $\begin{array}{l}0.34 \\
\pm 0.18\end{array}$ & - \\
\hline $\begin{array}{l}\text { Mikania } \\
\text { micrantha }\end{array}$ & - & - & - & - & - & - & - & - & $\begin{array}{l}0.16 \\
\pm 0.13 \\
\end{array}$ & $\begin{array}{l}3.20 \\
\pm 0.56 \\
\end{array}$ & $\begin{array}{l}20.0 \\
\pm 1.41 \\
\end{array}$ & - \\
\hline $\begin{array}{l}\text { Nitella } \\
\text { mucronatus }\end{array}$ & - & $\begin{array}{l}23.54 \\
\pm 1.53\end{array}$ & $\begin{array}{l}160 \\
\pm 4.00\end{array}$ & - & - & - & - & - & - & - & - & - \\
\hline $\begin{array}{l}\text { Polygonum } \\
\text { glabrum }\end{array}$ & - & - & - & - & - & - & - & - & - & - & - & $\begin{array}{l}0.6 \\
\pm 0.24 \\
\end{array}$ \\
\hline $\begin{array}{l}\text { Potamogeton } \\
\text { crispus }\end{array}$ & - & $\begin{array}{l}14.62 \\
\pm 1.21\end{array}$ & $\begin{array}{l}3.29 \\
\pm 0.53\end{array}$ & - & - & - & - & - & - & - & - & - \\
\hline $\begin{array}{l}\text { Spirodela } \\
\text { polyrhiza }\end{array}$ & $\begin{array}{l}1.66 \\
\pm 0.41 \\
\end{array}$ & $\begin{array}{l}6.53 \\
\pm 0.81 \\
\end{array}$ & $\begin{array}{l}2.53 \\
\pm 0.50 \\
\end{array}$ & $\begin{array}{l}2.30 \\
\pm 0.48 \\
\end{array}$ & $\begin{array}{l}2.45 \\
\pm 0.49 \\
\end{array}$ & $\begin{array}{l}3.04 \\
\pm 0.55 \\
\end{array}$ & $\begin{array}{l}1.88 \\
\pm 0.43 \\
\end{array}$ & $\begin{array}{l}7.80 \\
\pm 0.88 \\
\end{array}$ & $\begin{array}{l}4.64 \\
\pm 0.68 \\
\end{array}$ & $\begin{array}{l}3.76 \\
\pm 0.61 \\
\end{array}$ & $\begin{array}{r}12.94 \\
\pm 1.14 \\
\end{array}$ & $\begin{array}{l}7.6 \\
\pm 0.87 \\
\end{array}$ \\
\hline Total & 390.86 & 176.01 & 267.08 & 908.34 & 607.47 & 1097.64 & 1467.77 & 1398.72 & 884.53 & 1212.58 & 1350.69 & 967.0 \\
\hline
\end{tabular}

\section{Nutrients}

Nitrogen concentration in roots of E. crassipes was highest $(1.96 \%)$ in October and lowest $(1.26 \%)$ in June (Table 3). The concentration decreased gradually from November to March, had increasing-decreasing trend till June, thereafter increasing trend till October. On the other hand, nitrogen concentration increased in stems from 2.24\% in December to $2.89 \%$ in February, decreased gradually to $1.01 \%$ in June and thereafter increased gradually till October. Nitrogen concentration in whole leaves and pseudolaminae was maximum $(2.17 \%$ and $1.72 \%$, respectively) in December and minimum concentration of nitrogen $(0.62 \%)$ in whole leaves occurred in March, whereas in pseudolaminae minimum concentration $(0.21 \%)$ was recorded in August. Comparatively, nitrogen concentration in different parts of the plant occurred in the order: stems $>$ whole leaves $>$ roots $>$ pseudolaminae.

Phosphorus concentration in roots of E. crassipes ranged between 1 (Dec) and $1.75 \%(\mathrm{Sept})$ (Table 3). In stems, maximum $(0.65 \%)$ phosphorus concentration was recorded in October and minimum $(0.35 \%)$ in July. The phosphorus concentration decreased gradually from November to January, increased in February, had abrupt decrease in April, and increasing-decreasing trend till July. In whole leaves, the range of phosphorus concentration was between 0.35 (Aug and 
Jan) and $0.62 \%$ (March) whereas in pseudolaminae phosphorus concentration ranged between 0.2 (Jan) and $0.75 \%$ (Sept). There was increasing trend in phosphorus concentration from May to September and decreasing-increasing trend from October to March in all plant parts.

Potassium concentration ranged between 0.8 (Jan) and 1.8\% (Dec) in roots, between 0.7 (Aug) and $2.67 \%$ (Nov) in stems, between 0.7 (January) and $2.7 \%$ (April) in whole leaves, and between 0.88 (January) and $2.85 \%$ (October) in pseudolaminae of E. crassipes (Table 3). In roots, the concentration had decreasing trend from March to May, and thereafter increasingdecreasing trend till January. In stems, the concentration increased from 2.62 in March to 2.67\% in November. In whole leaves, the concentration had increasing-decreasing trend from June to January and an increasing trend from February to April. In pseudolaminae, potassium concentration had increasing trend from Feb. to June and decreasing trend from Nov. to Jan.

Musil and Breen (1977) reported 1.789, 2.171 and 4.99\% nitrogen; 0.309, 0.423 and $0.567 \%$ phosphorus; and $1.417,5.072$ and $2.817 \%$ potassium; respectively in roots, petioles and pseudolaminae of E. crassipes. Internal concentration of nitrogen, phosphorus and potassium considered adequate (to prevent deficiency symptoms) for higher plants is $1.5,0.2$, and $1 \%$, respectively (Salisbury \& Ross, 1992). Accordingly internal concentration of all these nutrients in $E$. crassipes was more than adequate throughout the year except nitrogen in pseudolaminae (Table 3). Although moderate excess of the nutrients than plant's requirements seems to offer no further advantage for growth, but it cannot be excluded that other ecologically important properties conferring competitive advantages- such as resistance to parasites or extreme climatic situations are, in fact, promoted (Larcher, 1975).

Table 3. Monthly variations in percentage concentrations of nitrogen, phosphorus and potassium in different parts of Echhornia crassipes (mean $\pm \mathrm{SE}$; $\mathrm{n}=3$ ).

\begin{tabular}{|c|c|c|c|c|c|c|c|c|c|c|c|c|}
\hline & \multicolumn{3}{|c|}{ Roots } & \multicolumn{3}{|c|}{ Stems } & \multicolumn{3}{|c|}{ Whole leaves } & \multicolumn{3}{|c|}{ Pseudolaminae } \\
\hline & $\mathbf{N}$ & $\mathbf{P}$ & $\mathbf{K}$ & $\mathbf{N}$ & $\mathbf{P}$ & $\mathbf{K}$ & $\mathbf{N}$ & $\mathbf{P}$ & $\mathbf{K}$ & $\mathbf{N}$ & $\mathbf{P}$ & $\mathbf{K}$ \\
\hline Jan & $\begin{array}{c}1.59 \\
\pm 0.72\end{array}$ & $\begin{array}{c}1.20 \\
\pm 0.63\end{array}$ & $\begin{array}{c}0.80 \\
\pm 0.50\end{array}$ & $\begin{array}{c}2.25 \\
\pm 0.86\end{array}$ & $\begin{array}{c}0.45 \\
\pm 0.38\end{array}$ & $\begin{array}{c}1.56 \\
\pm 0.72\end{array}$ & $\begin{array}{c}1.68 \\
\pm 0.74\end{array}$ & $\begin{array}{c}0.35 \\
\pm 0.33\end{array}$ & $\begin{array}{c}0.70 \\
\pm 0.47\end{array}$ & $\begin{array}{c}1.07 \\
\pm 0.59\end{array}$ & $\begin{array}{c}0.20 \\
\pm 0.24\end{array}$ & $\begin{array}{c}0.88 \\
\pm 0.53\end{array}$ \\
\hline Feb & $\begin{array}{c}1.54 \\
\pm 0.71 \\
\end{array}$ & $\begin{array}{c}1.45 \\
\pm 0.69 \\
\end{array}$ & $\begin{array}{c}1.60 \\
\pm 0.72 \\
\end{array}$ & $\begin{array}{c}2.89 \\
\pm 0.97 \\
\end{array}$ & $\begin{array}{c}0.60 \\
\pm 0.44 \\
\end{array}$ & $\begin{array}{c}2.62 \\
\pm 0.93 \\
\end{array}$ & $\begin{array}{c}2.19 \\
\pm 0.85 \\
\end{array}$ & $\begin{array}{c}0.57 \\
\pm 0.43 \\
\end{array}$ & $\begin{array}{c}2.60 \\
\pm 0.92 \\
\end{array}$ & $\begin{array}{c}1.56 \\
\pm 0.72 \\
\end{array}$ & $\begin{array}{c}0.45 \\
\pm 0.38 \\
\end{array}$ & $\begin{array}{c}2.70 \\
\pm 0.94 \\
\end{array}$ \\
\hline March & $\begin{array}{c}1.43 \\
\pm 0.68\end{array}$ & $\begin{array}{c}1.17 \\
\pm 0.62\end{array}$ & $\begin{array}{c}1.60 \\
\pm 0.72\end{array}$ & $\begin{array}{c}1.92 \\
\pm 0.80\end{array}$ & $\begin{array}{c}0.60 \\
\pm 0.44\end{array}$ & $\begin{array}{c}2.62 \\
\pm 0.93\end{array}$ & $\begin{array}{c}0.62 \\
\pm 0.44\end{array}$ & $\begin{array}{c}0.62 \\
\pm 0.44\end{array}$ & $\begin{array}{c}2.67 \\
\pm 0.94\end{array}$ & $\begin{array}{c}1.05 \\
\pm 0.59\end{array}$ & $\begin{array}{c}0.47 \\
\pm 0.38\end{array}$ & $\begin{array}{c}2.67 \\
\pm 0.94\end{array}$ \\
\hline April & $\begin{array}{c}1.49 \\
\pm 0.70 \\
\end{array}$ & $\begin{array}{c}1.15 \\
\pm 0.61 \\
\end{array}$ & $\begin{array}{c}1.40 \\
\pm 0.67 \\
\end{array}$ & $\begin{array}{c}1.14 \\
\pm 0.61 \\
\end{array}$ & $\begin{array}{c}0.42 \\
\pm 0.37 \\
\end{array}$ & $\begin{array}{c}2.50 \\
\pm 0.91 \\
\end{array}$ & $\begin{array}{c}1.98 \\
\pm 0.81 \\
\end{array}$ & $\begin{array}{c}0.52 \\
\pm 0.41 \\
\end{array}$ & $\begin{array}{c}2.70 \\
\pm 0.94 \\
\end{array}$ & $\begin{array}{c}0.94 \\
\pm 0.55 \\
\end{array}$ & $\begin{array}{c}0.41 \\
\pm 0.36 \\
\end{array}$ & $\begin{array}{c}2.65 \\
\pm 0.93 \\
\end{array}$ \\
\hline May & $\begin{array}{c}1.40 \\
\pm 0.67\end{array}$ & $\begin{array}{c}1.65 \\
\pm 0.74\end{array}$ & $\begin{array}{c}1.16 \\
\pm 0.61\end{array}$ & $\begin{array}{c}1.08 \\
\pm 0.60\end{array}$ & $\begin{array}{c}0.45 \\
\pm 0.38\end{array}$ & $\begin{array}{c}1.80 \\
\pm 0.77\end{array}$ & $\begin{array}{c}1.47 \\
\pm 0.70\end{array}$ & $\begin{array}{c}0.55 \\
\pm 0.42\end{array}$ & $\begin{array}{c}2.00 \\
\pm 0.81\end{array}$ & $\begin{array}{c}0.37 \\
\pm 0.34\end{array}$ & $\begin{array}{c}0.45 \\
\pm 0.38\end{array}$ & $\begin{array}{r}2.75 \\
\pm 0.95\end{array}$ \\
\hline June & $\begin{array}{c}1.26 \\
\pm 0.64 \\
\end{array}$ & $\begin{array}{c}1.25 \\
\pm 0.64 \\
\end{array}$ & $\begin{array}{c}1.56 \\
\pm 0.72 \\
\end{array}$ & $\begin{array}{c}1.01 \\
\pm 0.57 \\
\end{array}$ & $\begin{array}{c}0.38 \\
\pm 0.34 \\
\end{array}$ & $\begin{array}{c}1.56 \\
\pm 0.72 \\
\end{array}$ & $\begin{array}{c}1.52 \\
\pm 0.70\end{array}$ & $\begin{array}{c}0.50 \\
\pm 0.40 \\
\end{array}$ & $\begin{array}{c}2.55 \\
\pm 0.92 \\
\end{array}$ & $\begin{array}{c}0.96 \\
\pm 0.56\end{array}$ & $\begin{array}{c}0.47 \\
\pm 0.38 \\
\end{array}$ & $\begin{array}{c}2.75 \\
\pm 0.95 \\
\end{array}$ \\
\hline July & $\begin{array}{c}1.50 \\
\pm 0.70 \\
\end{array}$ & $\begin{array}{c}1.17 \\
\pm 0.62 \\
\end{array}$ & $\begin{array}{c}1.75 \\
\pm 0.76 \\
\end{array}$ & $\begin{array}{c}1.10 \\
\pm 0.60 \\
\end{array}$ & $\begin{array}{c}0.35 \\
\pm 0.33 \\
\end{array}$ & $\begin{array}{c}1.44 \\
\pm 0.69 \\
\end{array}$ & $\begin{array}{c}1.68 \\
\pm 0.74 \\
\end{array}$ & $\begin{array}{c}0.57 \\
\pm 0.43 \\
\end{array}$ & $\begin{array}{c}1.95 \\
\pm 0.80 \\
\end{array}$ & $\begin{array}{c}0.98 \\
\pm 0.56 \\
\end{array}$ & $\begin{array}{c}0.65 \\
\pm 0.45 \\
\end{array}$ & $\begin{array}{c}2.20 \\
\pm 0.85 \\
\end{array}$ \\
\hline Aug & $\begin{array}{c}1.59 \\
\pm 0.72 \\
\end{array}$ & $\begin{array}{c}1.50 \\
\pm 0.70\end{array}$ & $\begin{array}{c}1.00 \\
\pm 0.57\end{array}$ & $\begin{array}{c}1.50 \\
\pm 0.70\end{array}$ & $\begin{array}{c}0.37 \\
\pm 0.34\end{array}$ & $\begin{array}{c}0.70 \\
\pm 0.47\end{array}$ & $\begin{array}{c}1.19 \\
\pm 0.62 \\
\end{array}$ & $\begin{array}{c}0.35 \\
\pm 0.33\end{array}$ & $\begin{array}{c}0.92 \\
\pm 0.54\end{array}$ & $\begin{array}{c}0.21 \\
\pm 0.26\end{array}$ & $\begin{array}{c}0.46 \\
\pm 0.38\end{array}$ & $\begin{array}{c}1.25 \\
\pm 0.64\end{array}$ \\
\hline Sept & $\begin{array}{r}1.56 \\
\pm 0.72 \\
\end{array}$ & $\begin{array}{c}1.75 \\
\pm 0.76 \\
\end{array}$ & $\begin{array}{c}1.60 \\
\pm 0.72 \\
\end{array}$ & $\begin{array}{c}1.52 \\
\pm 0.70 \\
\end{array}$ & $\begin{array}{c}0.60 \\
\pm 0.44 \\
\end{array}$ & $\begin{array}{c}2.25 \\
\pm 0.86 \\
\end{array}$ & $\begin{array}{c}1.84 \\
\pm 0.78 \\
\end{array}$ & $\begin{array}{c}0.51 \\
\pm 0.41 \\
\end{array}$ & $\begin{array}{c}0.50 \\
\pm 0.40 \\
\end{array}$ & $\begin{array}{c}1.78 \\
\pm 0.76 \\
\end{array}$ & $\begin{array}{c}0.75 \\
\pm 0.50 \\
\end{array}$ & $\begin{array}{c}2.80 \\
\pm 0.96 \\
\end{array}$ \\
\hline Oct & $\begin{array}{c}1.96 \\
\pm 0.80\end{array}$ & $\begin{array}{c}1.42 \\
\pm 0.68\end{array}$ & $\begin{array}{c}1.56 \\
\pm 0.72\end{array}$ & $\begin{array}{c}1.98 \\
\pm 0.81\end{array}$ & $\begin{array}{c}0.65 \\
\pm 0.45\end{array}$ & $\begin{array}{c}2.62 \\
\pm 0.93\end{array}$ & $\begin{array}{c}1.57 \\
\pm 0.72\end{array}$ & $\begin{array}{c}0.51 \\
\pm 0.41\end{array}$ & $\begin{array}{c}2.57 \\
\pm 0.92\end{array}$ & $\begin{array}{c}1.17 \\
\pm 0.62\end{array}$ & $\begin{array}{c}0.60 \\
\pm 0.44\end{array}$ & $\begin{array}{c}2.85 \\
\pm 0.97\end{array}$ \\
\hline Nov & $\begin{array}{c}1.80 \\
\pm 0.77 \\
\end{array}$ & $\begin{array}{c}1.35 \\
\pm 0.67 \\
\end{array}$ & $\begin{array}{c}1.20 \\
\pm 0.63 \\
\end{array}$ & $\begin{array}{c}1.68 \\
\pm 0.74 \\
\end{array}$ & $\begin{array}{c}0.57 \\
\pm 0.43 \\
\end{array}$ & $\begin{array}{c}2.67 \\
\pm 0.94\end{array}$ & $\begin{array}{c}1.89 \\
\pm 0.79 \\
\end{array}$ & $\begin{array}{c}0.50 \\
\pm 0.40\end{array}$ & $\begin{array}{c}2.60 \\
\pm 0.92 \\
\end{array}$ & $\begin{array}{c}1.19 \\
\pm 0.62\end{array}$ & $\begin{array}{c}0.50 \\
\pm 0.40\end{array}$ & $\begin{array}{c}2.82 \\
\pm 0.96 \\
\end{array}$ \\
\hline Dec & $\begin{array}{c}1.66 \\
\pm 0.74\end{array}$ & $\begin{array}{c}1.00 \\
\pm 0.57\end{array}$ & $\begin{array}{c}1.80 \\
\pm 0.77\end{array}$ & $\begin{array}{c}2.24 \\
\pm 0.86\end{array}$ & $\begin{array}{c}0.50 \\
\pm 0.40\end{array}$ & $\begin{array}{c}2.04 \\
\pm 0.82\end{array}$ & $\begin{array}{c}2.19 \\
\pm 0.85\end{array}$ & $\begin{array}{c}0.47 \\
\pm 0.38\end{array}$ & $\begin{array}{c}2.62 \\
\pm 0.93\end{array}$ & $\begin{array}{c}1.72 \\
\pm 0.75\end{array}$ & $\begin{array}{c}0.60 \\
\pm 0.44\end{array}$ & $\begin{array}{c}2.77 \\
\pm 0.95\end{array}$ \\
\hline
\end{tabular}




\section{Water and mud}

The $\mathrm{pH}$ of water samples ranged between 6.26 and 6.4 being slightly more acidic in rainy and winter seasons than the summer season (Table 4). Temperature of water was higher in summer and rainy seasons than the winter season. The electrical conductivity of water samples ranged between 157 (rainy) and $586 \mathrm{ds} / \mathrm{m}$ (summer season). Both turbidity and dissolved solids were maximum (95 NTU and $650 \mathrm{mg} / \mathrm{l}$, respectively) during rainy season and minimum (7.13 NTU and $1.27 \mathrm{mg} / \mathrm{l}$, respectively) during winter season. Concentration of phosphorus was maximum $(0.49 \mathrm{mg} / \mathrm{l})$ in rainy and minimum $(0.07 \mathrm{mg} / \mathrm{l})$ in summer season, whereas concentration of nitrogen was maximum $(8.23 \mathrm{mg} / \mathrm{l})$ in winter and minimum $(3.26 \mathrm{mg} / \mathrm{l})$ in rainy season. Both ammonia and nitrate had maximum concentration $(1.16$ and $0.15 \mathrm{mg} / \mathrm{l}$, respectively) in summer season and maximum amount of nitrite $(0.05 \mathrm{mg} / \mathrm{l})$ was recorded in winter season. The concentration of potassium in water samples ranged between 4.3 (rainy) and $19.7 \mathrm{mg} / \mathrm{l}$ (winter). In general, water temperature was influenced by air temperature, whereas minimum values of nutrient parameters can be attributed to increase in water level and their utilization by live biomass; and maximum values to mineralization of organic matter and senescent phase of growth of E. crassipes.

Table 4. Physico-chemical properties of water in Eichhornia-Lemna-Spirodela association (mean $\pm \mathrm{SE} ; \mathrm{n}=3$ ).

\begin{tabular}{llll}
\hline & Winter $($ Jan $)$ & Summer (May) & Rainy (Sept) \\
\hline $\mathrm{pH}$ & $6.29 \pm 1.44$ & $6.40 \pm 1.47$ & $6.26 \pm 1.44$ \\
Temperature $\left({ }^{\circ} \mathrm{C}\right)$ & $17.20 \pm 2.39$ & $30.60 \pm 3.19$ & $31.20 \pm 3.22$ \\
Conductivity $(\mathrm{ds} / \mathrm{m})$ & $254.30 \pm 9.20$ & $586 \pm 13.97$ & $157 \pm 7.23$ \\
Turbidity $(\mathrm{NTU})$ & $7.13 \pm 1.53$ & $67 \pm 4.72$ & $95 \pm 5.62$ \\
Total dissolved solids $(\mathrm{mg} / \mathrm{l})$ & $127.40 \pm 6.51$ & $287 \pm 9.77$ & $650 \pm 14.71$ \\
Total nitrogen $(\mathrm{mg} / \mathrm{l})$ & $8.25 \pm 1.65$ & $4.37 \pm 1.20$ & $3.26 \pm 1.03$ \\
Ammonia $(\mathrm{mg} / \mathrm{l})$ & $0.27 \pm 0.30$ & $1.16 \pm 0.61$ & $1.12 \pm 0.60$ \\
Nitrate $(\mathrm{mg} / \mathrm{l})$ & $0.05 \pm 0.12$ & $0.15 \pm 0.22$ & $0.001 \pm 0.01$ \\
Nitrite $(\mathrm{mg} / \mathrm{l})$ & $0.05 \pm 0.12$ & $0.002 \pm 0.01$ & $0.001 \pm 0.01$ \\
Total phosphorus $(\mathrm{mg} / \mathrm{l})$ & $0.11 \pm 0.20$ & $0.07 \pm 0.14$ & $0.49 \pm 0.40$ \\
Potassium $(\mathrm{mg} / \mathrm{l})$ & $19.70 \pm 2.56$ & $6.40 \pm 1.45$ & $4.30 \pm 1.19$ \\
\hline
\end{tabular}

The $\mathrm{pH}$ of mud (base layer soil) of E. crassipes association was slightly more acidic in winter than summer and rainy seasons (Table 5). The proportionate composition of soil separates indicated silty-clay texture. Soil had the lowest concentration of nitrogen $(0.21 \%)$ in rainy, and phosphorus and potassium $(0.001$ and $0.0095 \%$, respectively) in winter; whereas the highest concentration of nitrogen $(0.29 \%)$ occurred in summer, phosphorus $(0.0016 \%)$ in summer and rainy, and potassium $(0.2001 \%)$ in rainy season. Comparatively, mud of the E. crassipes had higher concentration of potassium but lower concentrations of nitrogen and phosphorus than Typha domingensis association (Koirala et al., 2013). Percentage organic carbon in mud ranged 
between 3.6 and 4.3. This range of organic carbon is a characteristic of mineral soils that occur in freshwater marshes and riparian forests in which $\mathrm{pH}$ is circumneutral and nutrient availability is high (Mitsch \& Gosselink, 2000). In comparison to around the year grazed grassland at Biratnagar which has 14.5:1 C/N ratio (Jha, 2010), mud of E. crassipes association had 16:1 $\mathrm{C} / \mathrm{N}$ ratio (Table 5) due to accumulation of organic matter as a result of decreased decomposition in anaerobic environment.

Table 5. Seasonal variations in physico-chemical properties of mud (base layer soil) in Eichhornia-Lemna-Spirodelaassociation (mean $\pm \mathrm{SE}$; $\mathrm{n}=3$ ).

\begin{tabular}{llll}
\hline & Winter (Jan) & Summer (May) & Rainy (Sept) \\
\hline $\mathrm{pH}$ & $5.2 \pm 1.31$ & $4.7 \pm 1.24$ & $5.2 \pm 1.31$ \\
\hline $\begin{array}{l}\text { Texture }(\%) \\
\quad \text { Sand }\end{array}$ & & \\
$\quad$ Silt & $27.1 \pm 3.00$ & $30.7 \pm 3.19$ & $20.7 \pm 2.62$ \\
$\quad$ Clay & $47.7 \pm 3.98$ & $46.8 \pm 3.94$ & $52.8 \pm 4.19$ \\
\hline Nutrients $(\%)$ & $30.2 \pm 3.17$ & $22.5 \pm 2.73$ & $26.5 \pm 2.97$ \\
$\quad \mathrm{~N}$ & & & \\
$\quad \mathrm{P}$ & $0.25 \pm 0.28$ & $0.29 \pm 0.30$ & $0.21 \pm 0.26$ \\
$\quad \mathrm{~K}$ & $0.0011 \pm 0.02$ & $0.0016 \pm 0.02$ & $0.0016 \pm 0.02$ \\
\hline Organic carbon $(\%)$ & $4.30 \pm 1.19$ & $3.97 \pm 1.14$ & $3.60 \pm 1.09$ \\
\hline C/N ratio & $17.20: 1$ & $13.69: 1$ & $17.14: 1$ \\
\hline
\end{tabular}

In brief, findings of the present study will be helpful in better management of $E$. crassipes stands from both economic and ecological viewpoints.

\section{Acknowledgements}

Thanks are due to the National Agricultural Research Council (NARC), Khumaltar, Lalitpur and SEAM-N (Strengthening of the Environmental Administration and Management at the local level, Nepal), Biratnagar for lab facilities.

\section{References}

APHA (American Public Health Association) 1990. Standard Methods for the Examination of Water and Waste Water, $16^{\text {th }}$ edn. APHA, Inc., New York, USA.

Bellamy, D.J. 1967. Succession and the depth-time scale in ephemeral swamp ecosystem. Tropical Ecology 8: 67-73.

Cook, C.D.K. 1996. Aquatic and Wetland Plants of India. Oxford University Press, Oxford, UK.

Curtis, J.T. \& R.P. McIntosh 1951. An upland forest continuum in the prairie-forest border region of Wisconsin. Ecology 32: 476-498. http://dx.doi.org/10.2307/1931725 
Das, R.R. 1968. Growth and distribution of Eichhornia crassipes and Spirodela polyrhiza. Ph.D. Thesis, Banaras Hindu University, Varanasi.

Furman, N.H. (ed.). 1962. Standard Methods of Chemical Analysis, $6^{\text {th }}$ edn. D. Van Nostrand Co., New Jersey, USA.

Jha, S. 2010. Seasonal changes in physico-chemical properties of soil and forage nutrients in a lowland Nepalese grassland. Pakistan Journal of Plant Sciences 16(2): 51-63.

Koirala, U., S. Jha \& K.N. Ghimire 2013. Ecology of Typha domingensis Pers. Nepalese Journal of Biosciences 3: 1-8.

Krebs, C.J. 1972. Ecology: The Experimental Analysis of Distribution and Abundance. Harper and Row, New York, USA.

Kroll, R.W., J.F. Gottgens \& B.P. Swartz 1997. Wild rice to rip-rap: 120 years of habitat changes and management of a Lake Erie coastal marsh. Transactions of the $62^{\text {nd }}$ North American Wildlife and Natural Resources Conference 62: 490-500.

Larcher, W. 1975. Physiological Plant Ecology. Springer-Verlag, New York, USA. http://dx.doi.org/ 10.1007/978-3-642-96281-3

McCann, K.S. 2000. The diversity-stability debate. Nature 405: 228-233. http://dx.doi.org/ $10.1038 / 35012234$

Mitsch, W.J. \& J.G. Gosselink 2000. Wetlands. John Wiley \& Sons, Inc., New York, USA

Musil, C.F. \& C.M. Breen 1977. The influence of site and position in the plant community on the nutrient distribution in and content of Eichhornia crassipes (Mart.) Solms. Hydrobiologia 53: 67-72. http://dx.doi.org/10.1007/BF00021233

Parija, P. 1934. Physiological investigations on water-hyacinth (Eichhornia crassipes) in Orissa with notes on some other aquatic weeds. Indian Journal of Agricultural Science 4: 399-429

Penfound, W.T. \& T.T. Earle 1948. The biology of water-hyacinth. Ecological Monographs 18: 447-472. http://dx.doi.org/10.2307/1948585

Piper, C.S. 1944. Soil and Plant Analysis. The University of Adelaide, Australia.

Salisbury, F.B. \& C.W. Ross 1992. Plant Physiology, $4^{\text {th }}$ edn. Wadsworth, Belmont, USA

Shannon, C.E \& W. Weaver 1949. The Mathematical Theory of Communication. University of Illinois Press, Urbana, USA.

Simpson, E.H. 1949. Measurements of diversity. Nature 163: 688. http://dx.doi.org/10.1038/163688a0

Singh, J.S. \& P.S. Yadava 1974. Seasonal variations in composition, plant biomass and net primary productivity of tropical grassland at Kurkshetra, India. Ecological Monographs 44: 351-376. http://dx.doi.org/10.2307/2937034

Walkley, A.J. \& I.A. Black 1934. An examination of method for determining organic carbon and nitrogen in soil. Journal of Agricultural Science 25: 589-609.

Willard, H.H., L.L Merritt \& J.A. Dean 1974. Instrumental Methods of Analysis $5^{\text {th }}$ edn. D. Van Nostrand Co., New Jersey, USA. 